\title{
ACID PRODUCTION OF ORAL BACTERIA ASSOCIATED WITH DENTAL CARIES ${ }^{1}$
}

\author{
MARSHALL L. SNYDER AND JUNE J. TEACHOUT \\ Hygienic Laboratory, University of Michigan, Ann Arbor, Michigan
}

A controversy still exists over the organism or organisms of the oral flora responsible for the acid that must be produced to decalcify the enamel and dentin in carious lesions. One group (1-6) holds that a single species or type (L. acidophilus) which not only can produce but also maintain an acid environment sufficient for in vivo decalcification ( $\mathrm{pH} 5.0$ ) is primarily responsible. Others (7-11) feel that the action is essentially non-specific and depends on the acidogenic powers of any one or combination of bacteria. Hence, it was thought of interest to report some simple experiments dealing with the aciduric and acidogenic properties of strains of oral lactobacilli, yeasts, streptococci, and staphylococci, which are the organisms most commonly associated with dental caries.

\section{MATERIAL AND METHODS}

For our purposes 30 strains each of yeasts and smooth lactobacilli were isolated by plating on tomato juice agar specimens of saliva from children showing clinically active caries. In a similar manner, employing blood agar, 30 strains each of streptococci and staphylococci were obtained from specimens of saliva of children with or without evidence clinically of caries activity. The streptococci were about equally divided between the alpha and gamma types. The majority of the staphylococci was without pigment. No attempt was made to use a selective medium such as described by Harrison (12) to facilitate the isolation of acidogenic streptococci. However, later trials with this medium indicated that acidogenic streptococei were not especially numerous in the samples of saliva cultured.

\section{EXPERIMENTAL}

The first experiment was designed to test the effect of acidity upon the growth of staphylococci, streptococci, and lactobacilli on tomato juice agar which is ordinarily used for estimating the number of lactobacilli in the saliva as an index of caries activity. Eighteen hour glucose broth cultures of the organisms were used and 1 loopful transferred to a tomato agar plate of a series adjusted with lactic acid or sodium hydroxide to $\mathrm{pH} 4.5,4.7,5.0,5.3$, and 5.5 as determined by the quinhydrone electrode. The plates were incubated at $37 \mathrm{C}$. for 96 hours and results are recorded in fig. 1 .

Fig. 1 indicates that while most of the strains of streptococci grew readily

1 Received for publication May 26, 1942. 
at $\mathrm{pH} 5.5$, only 2 were found to grow at $\mathrm{pH}$ 5.3. All the strains of staphylococci grew at $\mathrm{pH} 5.0$ and the majority at $\mathrm{pH} 4.7$. Not until $\mathrm{pH} 4.5$ was reached was there a marked diminution in the growth of the staphylococci, but 25 of the 30 strains of lactobacilli were still capable of definite growth at this hydrogen-ion concentration.

The second experiment sought to obtain some information not only about the production of acid sufficient for decalcification ( $\mathrm{pH} \mathrm{5.0)}$ but also what influence this acidity had upon the viability of the organisms. All 30 strains of yeast, lactobacilli, staphylococci, and streptococci were inoculated respectively into tubes of glucose broth. Viability was tested by removal of 1 loopful from each culture to fresh glucose broth at 24 hour intervals for 10 days. The appearance of turbidity in the subculture by bacteria morphologically similar to the parent culture was considered evidence of growth. Acidity of the culture

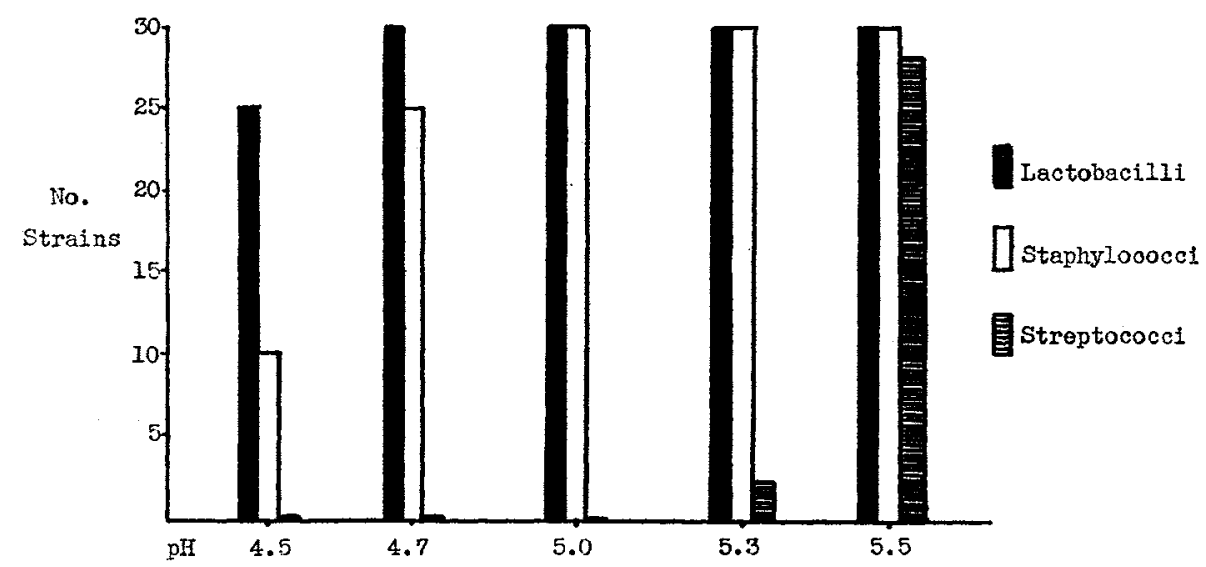

FIG. 1. Influence of acidity on growth of lactobacilli, staphylococci and streptococci on tomato juice agar

on the tenth day was determined by use of a spot plate with brom-cresol-green and brom-phenol-blue as the indicators. The data are listed in Table I.

It is obvious that the streptococci rapidly died out under the test conditions; only 2 strains survived for 10 days. In contrast all the other cultures grew readily after 10 days in the glucose broth. In respect to acid production only the lactobacilli were able to lower markedly the hydrogen-ion concentration below $\mathrm{pH} 5.0$, although the streptococci formed singly or on the average slightly more acid than the staphylococci and yeasts.

A portion of this experiment was later repeated by one of us (M. L. S.) using the serum glucose agar described by Harrison (12) which was modified only by the substitution of beef for veal infusion and horse for sheep serum. Specimens of saliva were obtained from 20 children of whom half were clinically negative for caries activity. A loopful of each specimen was streaked on a plate of the medium and 2 strains of streptococci were isolated from each plate before placing in glucose broth; the acidogenic types were identified by their 
yellow color or yellow zones around the colonies on the medium, and they were about equally represented with the non-acidogenic types for study. The glucose broth cultures were inoculated and incubated for 10 days at $37 \mathrm{C}$. after which a loopful was transferred to fresh glucose and serum dextrose agar. Although growth occurred in all but 3 tubes of the first series of glucose broth inoculated,

TABLE I

Acid production and viability of oral streptococci, staphylococci, yeasts and lactobacilli in 2 per cent glucose infusion broth

\begin{tabular}{|c|c|c|c|c|c|c|}
\hline \multirow{2}{*}{ IYPES } & \multirow{2}{*}{$\begin{array}{l}\text { No. } \\
\text { STRAINS }\end{array}$} & \multicolumn{4}{|c|}{ SORVIVAI IN DAYS } & \multirow{2}{*}{$\begin{array}{l}\text { AVERAGE } \\
\text { FINAL pH }\end{array}$} \\
\hline & & 1 & 2 & 5 & 10 & \\
\hline Streptococci. & 30 & 25 & 8 & 4 & 2 & 4.6 \\
\hline Staphylococci. & 30 & 30 & 30 & 30 & 30 & 4.7 \\
\hline Yeasts...... & 30 & 30 & 30 & 30 & 30 & 5.0 \\
\hline Lactobacilli. & 30 & 30 & 30 & 30 & 30 & 3.8 \\
\hline
\end{tabular}

TABLE II

Acid production in Brom-cresol-green Dextrose Agar by pure cultures and mixtures of $\$ 0$ strains each of oral streptococci, staphylococci, yeasts and lactobacilli

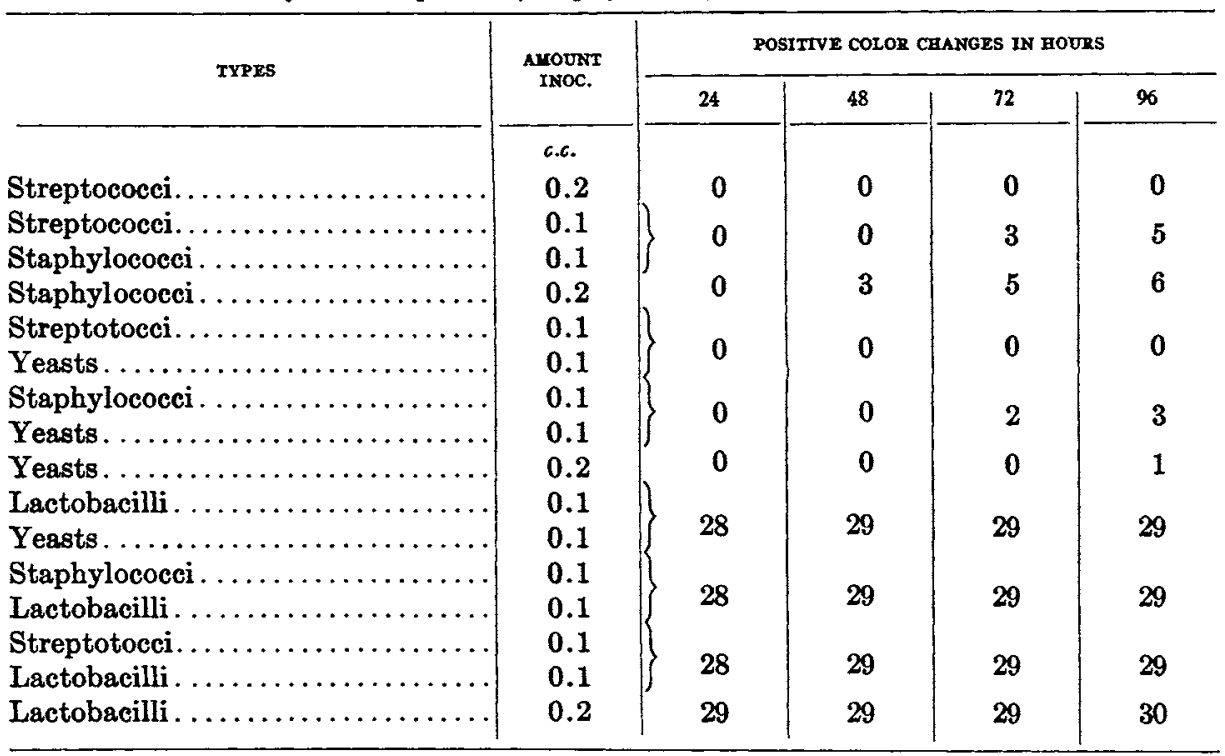

no growth was observed in the second series of glucose broth or on the serum glucose agar plates.

Since Fosdick and Hansen (13) have already shown the marked increase of lactic acid by lactobacilli in the presence of yeast, a third experiment was developed to test the synergistic or symbiotic behavior of these cultures. Glucose agar, ( $\mathrm{pH} 4.75),{ }^{2}$ containing brom-cresol-green as the indicator was used instead

${ }^{2}$ Supplied by the Difco Laboratories as Brom-Cresol-Green Dextrose Agar. 
of glucose broth. This medium has the advantage of dispersing the bacteria of the inoculum when used as a "shake" culture and allowing easy observation. It has also been found to be as satisfactory as tomato juice agar as a laboratory method in the diagnosis of caries activity (14). Tubes containing the agar were melted, cooled to $50 \mathrm{C}$, and inoculated either with $0.2 \mathrm{cc}$. of 18 hour glucose broth cultures of the various organisms or with $0.1 \mathrm{cc}$. of one culture and $0.1 \mathrm{cc}$. of another. In this way the effects of pure cultures and the action of one species in the presence of another was tested. The tubes were rotated after inoculation to mix the contents, after which they were allowed to solidify. Incubation was carried out at $37 \mathrm{C}$. for 96 hours with daily observation. Changes in the bluegreen color were considered positive only when green was no longer the dominant color and yellow took its place. The results are given in Table II. Table II is essentially self-explanatory. Neither the yeast nor the streptococci by themselves or in combination produced marked amounts of acid in 96 hours; a few strains of staphylococci did in 48 hours, but 29 of 30 strains of lactobacilli turned the medium yellow in 24 hours.

\section{DISCUSSION}

It is generally accepted that the essential mechanism of decalcification depends not only on the production by bacteria of acid in localized areas but also on the tolerance of the environment by the organisms concerned. The first part of the action has been demonstrated on many occasions and for several types of bacteria, as a result of which divergent claims for responsibility exist. References to the second requirement are not so frequent, and in the matter of actual in vivo decalcification little information is available, although the methods of Stephan (15) and Miller and Muntz (16) should be very helpful.

Our experiments were in vitro tests, and as such are open to every criticism applicable to these procedures. They do, however, emphasize once again the acidogenic and aciduric properties of the lactobacilli, which alone of the group produced on the average a hydrogen-ion concentration markedly below $\mathrm{pH} 5.0$. Although the terminal acidities in glucose broth of the other organisms reached this "critical" level for decalcification or slightly below, it is difficult to assume that in the face of the neutralizing factors in the mouth these would have quantitatively sufficient ions left for the necessary calcium exchange as compared with the lactobacilli.

In a similar manner the susceptibility of the oral streptococci to acid whether of their own metabolism or in a controlled environment was illustrated. It is extremely doubtful if the rate of acid formation, which has been shown by Fosdick and Starke (17) to be considerably more rapid for yeasts and streptococci than lactobacilli is anywhere near as important in decalcification as the ability to live or reproduce in surroundings inimical to their survival. This feature may explain the failure of Miller, Muntz and Bradel (18) to obtain significant differences in the formation of lactic acid by plaque material from carious and non-carious teeth; that is, the 30 minute fermentation period of the glucose by dried plaque material only showed the fermentation by the strepto- 
cocci which are numerically the dominant organism in the mouth and did not indicate the slower acting lactobacilli.

There is little or no question about the growth or survival of yeasts in an acid medium. Our strains were no exception. They produced consistently a pH of 5.0 in glucose broth, lived for 10 days in these cultures, and grew luxuriantly on the surface of the color medium. Fosdick and Wessinger (19) have reported a careful quantitative analysis of the fermentation of glucose by an oral strain of Saccharomyces cerevisiae, and have also shown the influence of this organism on lactic acid production by the lactobacilli. From these results they have postulated on the possible symbiotic role of the yeasts in dental caries. This theory is very attractive not only chemically but also because these forms are very frequent in carious mouths. However, there is one marked shortcoming: many, if not the majority, of these forms in the saliva are not true yeasts but are monilias (Knighton (20)). In our group of 30 strains only 2 formed ascospores on carrot plugs and could be considered belonging to Saccharomyces; the remaining strains produced soft creamy colonies on Sabouraud's medium with a tendency toward forming mycelia, features which would tend to classify them among the monilias rather than the yeasts.

Some interesting observations were made with the staphylococci of which a few strains showed definite aciduric tendencies as measured by color changes and survival in acid broth. Little attention has been paid to the staphylococci since they seem to be numerically unimportant in the salivary flora, but Snyder (21) previously pointed out that based on correlation coefficients the staphylococci approached more closely to significant figures $(0.3)$ than either the streptococci or yeasts.

At this time an experimental approach to the problem of symbiotic or synergistic action of these forms was tried, which might test the thesis of nonspecificity held by many. The results certainly offer no conclusive evidence, but they did show that outside of the lactobacilli only a few strains of staphylococci were able to produce positive color reactions in the brom-cresol-green dextrose agar. On the other hand, no information was secured by this method whether the amount of acid produced by combinations of lactobacilli and the other organisms was greater than that formed by the lactobacilli alone.

\section{SUMMARY}

The acidogenic and aciduric properties of 30 strains each of oral lactobacilli, streptococci, staphylococci, and yeast-like organisms were tested. All strains produced a terminal $\mathrm{pH}$ of 5.0 or less in glucose broth, but the lactobacilli alone lowered the hydrogen-ion concentration markedly below this level. On the basis of subculture after 10 days in glucose broth all except the streptococci could be considered aciduric; only 2 of the 30 strains were capable of being subcultured at the end of this period. In brom-cresol-green dextrose agar ( $\mathrm{pH} 4.75$ ) neither the streptococci nor yeast alone or in combination changed the color in 4 days. A few strains of staphylococci gave positive reactions in 48 hours.

However, 29 of the 30 strains of lactobacilli turned the medium in 24 hours or 
less. Symbiotic mixtures gave no indication of increased acid production under the conditions of the experiment.

\section{REFERENCES}

1. Rodriguez, F. E., J. A. D. A., 17: 1711, 1930.

2. Bunting, R. W., Hadley, F. P., JAY, P., ANd HARd, D. C., Am. Jour. Dis. Child., 40: $536,1930$.

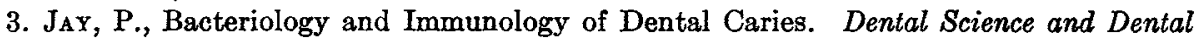
Art., Gordon, S. editor (Phila., Lea and Febiger; 1938).

4. JAY, P., Am. Jour. Pub. Health., 28: 759, 1938.

5. Enright, J. J., Frimseld, H. E., And Trescher, J. D. Res., 12: 759, 1932.

6. Johnston, M. M., Williams, C. H. M., Anderson, P. G., Drake, T. G. H., Tisdali, F. F., AND KAAKE, M. J., J. A. D. A., 23: 1493, 1936.

7. Anderson, T. G. AND Rettger, L. F., J. D. Res., 16: 489, 1937.

8. Tunnicliff, R. and Hammond, C., J. A. D. A., 25: 1047, 1938.

9. Clarke, J. K., Brit. J. Exp. Path., Б: 141, 1924.

10. Stream, L. P., D. Items Interest, 61: 1038, 1939.

11. Belding, P. H. ANd Belding, L. J., D. Items Interest, 62: 303, 1940.

12. Harrison, R. W., J. Inf. Dis., 65: 142, 1939.

13. Fosdick, L. S. And HANSen, H. L., J. A. D. A., 23: 401, 1936.

14. SNYDER, M. L., J. A. D. A., 28: $44,1941$.

15. Stephen, R. M., J. A. D. A., 27: 718, 1940.

16. Miller, B. F. ANd Muntz, J. A., J. Biol. Chem., 126: 413, 1938.

17. Fosdick, L. S. ANd Stark, A. C., J. A. D. A., 28: 234, 1941.

18. Mrller, B. F., Muntz, J. A., ANd Bradel, S., J. D. Res., $19: 473,1940$.

19. Fosdick, L. S. ANd Wessinger, G. D., J. A. D. A., $27: 203,1940$.

20. Knighton, H., J. D. Res., 18: 103, 1939.

21. SNTDER, M. L., J. D. Res., 18: 497, 1939. 